\title{
Co-creating value in research: stakeholders' perspectives
}

\section{A Centre for Research Excellence reflects on collaborating with policymakers and front-line staff to bring evidence into practice}

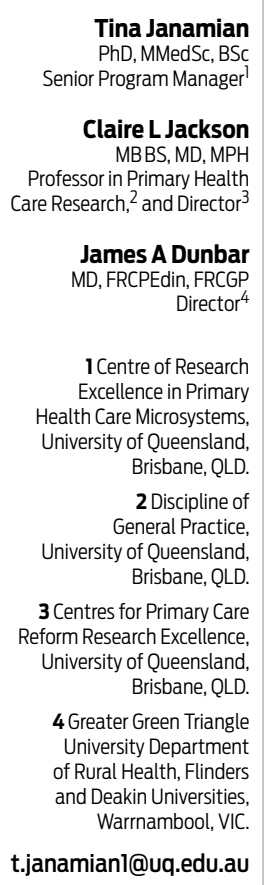

doi: $10.5694 / \mathrm{mjal} 4.00273$

Online first 21/07/14 ast year's Strategic review of health and medical research - better health through research (the McKeon Review)

highlighted strengthened partnerships between researchers, health care professionals, government and community as central to a productive research sector. ${ }^{1}$ Our Centre for Research Excellence (CRE) has, from inception, adopted such an approach to facilitate translation of our research into evidence-based health care and policy. ${ }^{2}$ Implementation research works best when the research partners who make policy or deliver care have been involved at all stages of the research process - from the research question through to the analysis and implementation strategy. ${ }^{3,4}$ In using these approaches, our CRE has partnered and worked closely with a variety of influential primary care organisations (Box). Collectively, they represent members delivering more than 100 million patient consultations per year or organisations responsible for setting the quality and safety benchmark for Australian primary care.

From 2011, our CRE has had regular "linkage and exchange" 5,6 activity with all partner organisations. This includes a variety of different communication methods — regular full-day research planning meetings, providing opportunities to receive updates and provide feedback, and informal input and advice around the CRE's research program. Our partner organisations also sit on our Research Stream Committees and National Advisory Committee, providing them with more formal opportunities to engage with the research team. As our partners reflect the heterogeneity of the end users of our research, this allows them to influence new approaches, generate fresh ideas and provide advice on existing frameworks.

In this article, we record insights from these nine organisations about their experience in working with our Centre. Our information was obtained from senior organisational representatives in open-ended telephone interviews, either individually or in small groups. Three key areas were explored: general perceptions about the partnership to date, the benefits and challenges of coproduction and implementation, and thoughts on future initiatives of this kind.

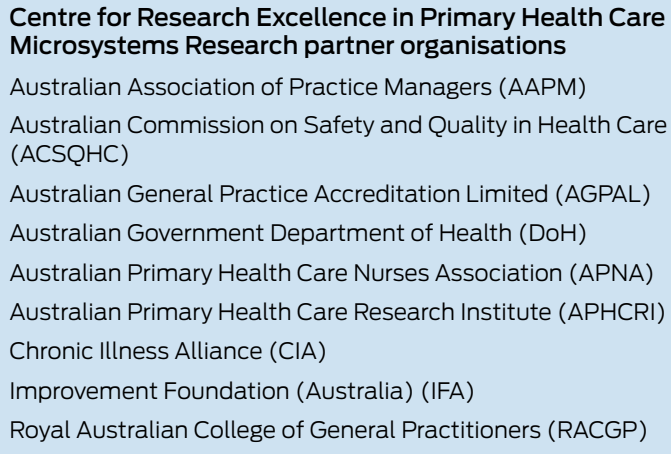

A journalist independently recorded the representatives' responses, and the transcriptions of these are included in this reflection piece.

\section{Benefits of the collaboration}

Most key informants perceived the collaboration to be value-adding and enriching.

Partnering with the CRE has helped us identify new opportunities and has added credibility to the quality frameworks we have in place. The ideas the Centre has brought to the table have developed our thinking. We have been able to consider and incorporate their quality improvement activities into our existing processes to add value to what we do. The open communication channels have been excellent throughout the process. (RACGP)

Most partners reported the collaborative relationship as respectful and open.

There has been good communication from the research team back to us, and the research has been professionally led and well managed. (AGPAL)

The partners were pleased to see the CRE bringing together key stakeholders from a range of disciplines to work together towards a common goal:

The thing that I've particularly valued has been the broader meetings that we've held at various times. To be able to hear people from a huge range of disciplines and perspectives actually sharing with one another and working towards a shared goal. (CIA)

The research team perceived working closely with the organisational end users to have been highly beneficial to the CRE research program. It allowed fine-tuning of research to produce outcomes implementable by partner organisations, particularly coproduction of research questions designed to generate the product identified by partners.

Partners identified issues of relationship, pragmatism and relevance as important.

The relationship is the important thing, and its what's been missing in other forms of what you might call applied research where a researcher goes away, undertakes the research and then perhaps publishes a paper, which then misses the bit as to "how do we get it implemented" ... This change from the top-down focus of previous programs could result in fewer failures and more successes and, generally speaking, better value for money. (IFA)

We wanted research that is practical, with commonsense outcomes that people can understand. That is what we have achieved ... and we recognise there will be useful outcomes. (AGPAL) 
Partnership has permitted progressive road testing of processes, findings and recommendations, which has been invaluable. Partners appreciate that the CRE conducts research that is practical, relevant, and will produce outcomes that can be operationalised by partner end users almost immediately.

A lot of the research that's normally done on the policy level is at a macro level — good ideas but vary rarely implemented in practice. People on the frontline are actually having significant input to the process and being able to inform the way that the practice improvement tool is developed. I think that's really important. (AAPM)

The partnership with the CRE has enabled us to actually have discussions so that we can make clear what are the needs of the organisations to enable them to make change, and the CRE gets involved in taking that on board in their research to indicate what might work best to help with patient safety improvement. We've also been working with the CRE in helping identify the best practices throughout Australia so we can work more closely and find out what makes them the best practices. By and large, it's been a process that has enabled us to have communication with the researching body who have worked with us in identifying the needs, the topics and of course now are involved in an interaction in how it might be implemented within our improvement framework. (IFA)

Further benefits relate to the CRE assisting stakeholders to improve quality and build accreditation options. Involvement in our research program has offered an opportunity to gain quality improvement and continuing professional development points through the RACGP, and, more recently, professional development points for practice managers through the AAPM.

It assists in their accreditation. They've got a system in place that will help them with continuous improvement and that does flow onto patient care and safety. It has empowered the practices to make changes towards quality improvement. (AAPM)

The CRE has found access to the partner organisations' membership and networks for recruitment to its research program invaluable. Through these, we have successfully engaged hundreds of practices across Australia to participate in our research program. This helps develop research capacity in primary health care organisations and practices and is consistent with both APHCRI's mandate and the McKeon Review, which states that "research capacity among health professionals is critical for conducting research, promoting research translation and improving the health system". ${ }^{1}$ Most partner organisations raised these benefits, and noted that the CRE has promoted involvement of the entire practice and primary care staff, not only general practitioners.

Previously, I think, people have thought it's really the physician's role and nothing to do with the office staff at all but this [CRE] has made it really clear that it's the responsibility of the whole team and shows how they

can all contribute to the end result which, of course, is patient safety ... people who have participated in the research have found it beneficial to their practices and to them personally. (AAPM)

It is well documented that only a fraction of research is translated to policy and practice. ${ }^{7}$ Our Centre addresses this gap by involving influential partners in both the creation and dissemination of evidence-based research into policy and practice. ${ }^{8}$ We have regularly engaged with policymakers to ensure that our research production aligns with their needs for easy uptake into policy and service delivery. It is pleasing that this style is welcomed by government, but is an area that requires more work.

\section{This change from the top- down focus of previous programs could \\ This relationship with the CRE reflects the broader move in government to have evidence at the centre of policy making ... whilst we articulate that as an ideal, we are still in the process of determining how we do that. $(\mathrm{DoH})$} result in ... more successes and, generally speaking, better value for money

\section{Challenges of the collaboration}

Working across the research-policy interface often poses challenges related to differing cultures, priorities, preferred style of communication, and time frames.

The research method takes years to produce its outputs and the questions that policymakers seek answers on don't always coincide with the time scales in the research ... the challenge that we face is to how to articulate the policy need and the importance of this research in the absence of outputs. (APHCRI)

The policy-making process is not necessarily a linear process that happens in discrete time frames. The capacity for the CRE to work with us in that environment is going to be different at times for the CRE where they want to start a project and progress through in a neat timeframe. $(\mathrm{DoH})$

It was acknowledged that it takes commitment and time to build relationships and address cultural differences.

It is early stages and is still a maturing relationship. I think from both sides, there are different cultures and different contexts in which we work. It takes time to actually build that relationship up and understand those different contexts, and for two different cultures to come together to do something new. $(\mathrm{DoH})$

When asked regarding enhanced collaboration, several partners felt that their involvement was limited and would have preferred to be engaged more intensively:

To continue involvement we want to be kept involved at a higher level. There is the quarterly magazine, APNA annual conference ... it is a great opportunity for the CRE and really good for APNA to be able to let people know what we are involved in. (APNA)

It has worked well, but sometimes, I feel disconnected from it. That is probably largely from the Commission end rather than the CRE end. If we were starting again I would push harder to be more involved. (ACSQHC) 


\section{The future}

APHCRI CREs have unique assets in staff and skills linked to extensive national and international networks for primary care capacity building.

It would be useful to extend some of this preliminary work. Good primary health care has a positive impact on the overall health system and research is essential to achieving those improvements. (RACGP)

Most partners felt that it has taken significant time and energy to build the relationships required to effectively conduct research in partnership, but there is much work yet to be done.

We've brought them to a level where they're working well together and delivering ... so how can we maintain these relationships? It often is reliant on the personal relationships that have been built up. (CIA)

I think it has been good in terms of starting that relationship, working together and a willingness to cooperate but I don't think we've got the fullness of maturity yet that we would like. $(\mathrm{DoH})$

All informants recognised the importance of the CRE in building "grassroots" capacity to deliver practical solutions for improving patient safety, clinical outcomes and overall quality of care, and indicated an interest in continuing their involvement in research development of this nature. The interlocking of end user and researcher across the entire Centre research journey has taken time, patience and flexibility on both sides. Yet the benefits in terms of research translation and utility have already been significant, and are documented in each paper in this Supplement. Research end users, are critical and willing partners in closing the primary care evidence-practicepolicy loop, for the benefit of all Australian communities.

Acknowledgements: The research reported in this article is a project of the APHCRI, which is supported by a grant from the Australian Government Department of Health and Ageing. The information and opinions contained in it do not necessarily reflect the views or policy of the APHCRI or the Department. We thank the following representatives from our partner organisations who participated in an interview: Mark Booth (First Assistant Secretary, Primary and Ambulatory Care Division, DoH), Chris Carlile (Assistant Secretary, Policy, Performance and Quality Branch Primary and Ambulatory Care Division, DoH), and Hope Peisley (Director, Research and Quality Section, Policy, Performance and Quality Branch Primary and Ambulatory Care Division, DoH); Peter McInnes (APHCRI/DoH Knowledge Broker), Mike Civil (Chair, National Standing Committee - Standards for General Practices, RACGP) and Josephine Raw (General Manager, Policy, Practice and Innovation, RACGP); Stephen Clark (CEO, AGPAL and Quality Innovation Performance), Gillian Leach (CEO, AAPM), Dale Ford (Principal Clinical Adviser, IFA); Christine Walker (CEO, CIA), Julianne Badenoch (President, APNA) and Nicola Dunbar (Director, Strategy and Development, ACSQHC).

Competing interests: No relevant disclosures.

Provenance: Commissioned; externally peer reviewed.

1 Australian Government Department of Health and Ageing. Strategic review of health and medical research. Better health through research. Canberra: Commonwealth of Australia, 2013. http://www.mckeonreview. org.au/downloads/Strategic_Review_of_Health_and_Medical_Research_ Feb_2013-Final_Report.pdf (accessed Dec 2013).

2 Jackson CL, Janamian T, van Weel C, Dunbar JA. Implementation research - its importance and application in primary care. Med J Aust 2014; 201 (3 Suppl): S42-S43.

3 May CR, Mair F, Finch T, et al. Development of a theory of implementation and integration: Normalization Process Theory. Implement Sci 2009; 4: 29.

4 Ramaswamy V, Ozcan K. The co-creation paradigm. Redwood City, Calif: Stanford University Press, 2014.

5 Lomas J, Brown AD. Research and advice giving: a functional view of evidence-informed policy advice in a Canadian Ministry of Health. Milbank Q 2009; 87: 903-926.

6 Choi BC, Pang T, Lin V, et al. Can scientists and policy makers work together? J Epidemiol Community Health 2005; 59: 632-637.

7 Green LW, Ottoson JM, García C, Hiatt RA. Diffusion theory and knowledge dissemination, utilization, and integration in public health. Annu Rev Public Health 2009; 30: 151-174.

8 Brownson RC, Colditz GA, Proctor EK. Dissemination and implementation research in health: translating science to practice. New York: Oxford University Press, 2012 